\title{
A Confocal Scanning Optical Microscope System for Measuring Refractive Index Profiles of Specialty Optical Waveguides
}

\author{
Youngchun Youk and Dug Young Kim
}

Department of Information and Communications, Gwangju Institute of Science and Technology, 1 Oryongdong, Bukgu, Gwangju, 500-712, South Korea

Various methods to measure the refractive index profiles of optical waveguides and optical fibers have been reported ever since the development of index guided optical fibers and waveguides. Refracted near-field (RNF) method, transverse interferometric (TM) method, and transmitted nearfield (TNF) method are conventional methods used for this purpose [1]. However these methods have a critical drawback that a sample fiber or a waveguide needs to be immersed in an index matching oil and a measured refractive index profile is affected by the index of the index matching oil itself.

In this paper, we demonstrate a highly repeatable refractive index profile measurement technique using a modified confocal scanning optical microscope. As this method measures reflectivity change due to refractive index change on a waveguide facet [2] it does not require index matching oil and it can be applicable to waveguides with complex and axially nonsymmetric structure. Even though several confocal microscope systems had been used to measure refractive index profiles by several research groups [3-5], these approaches were not very successful because of problems such as laser instability and surface imperfections. By using a stable LED source, low signal detection system and simple feedback system we have obtained a good refractive index precision and repeatability.

Fig. 1 and Fig. 2 show the refractive index measurements of two specialty optical fibers. Fig. 1(a) shows the measured refractive index profile of a step index multimode fiber with $13.4 \mu \mathrm{m}$ core size and 0.0328 peak refractive index difference. The measurement scan range is $30 \mu \mathrm{m} \times 30 \mu \mathrm{m}$ with a step size of $300 \mathrm{~nm}$. Fig. 1(b) shows the comparison between our measurement result for the sample fiber and the refractive index of its preform measured with a conventional preform analyzer.

Fig. 2(a) shows the cross-sectional image of a Polarizing Maintaining Fiber (PMF). Fig. 2(b) shows the measured refractive index profile of a PMF, and Fig. 2(c) shows its contour plot

\section{References}

[1] D. Marcuse, Principles of Optical Fiber Measurement, Academic Press, New York, 1981.

[2] Y. Park et al., Meas. Sci. Technol., 13 (2002) 1.

[3] W. Eickhoff and E. Weidel, Opt. Quantum Electron., 7 (1975) 109.

[4] M. Ikeda et al., Appl. Opt., 14 (1975) 814.

[5] M. Tateda, Appl. Opt., 17 (1978) 475.

[6] This work was supported in part by the Korea Science and Engineering Foundation through the Ultra-Fast Fiber-Optic Networks Research Center, and the Korean Ministry of Education \& Human Resources Development through the Brain Korea 21 Program. 


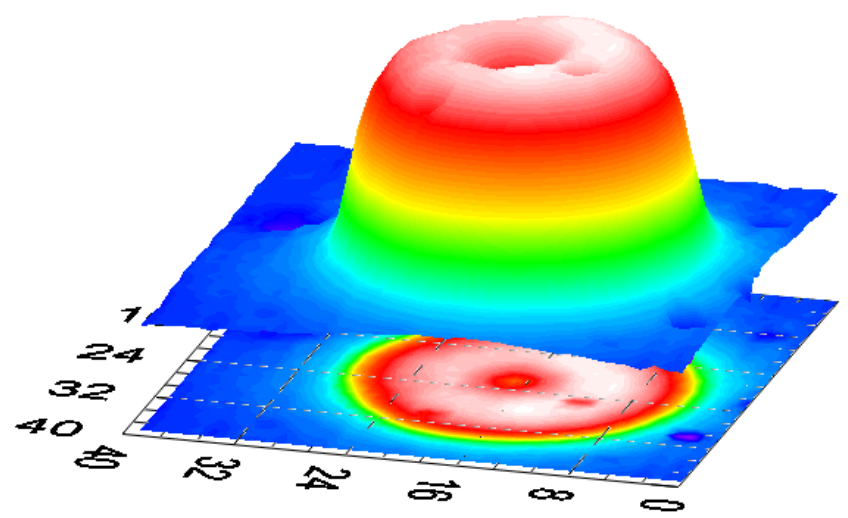

(a)

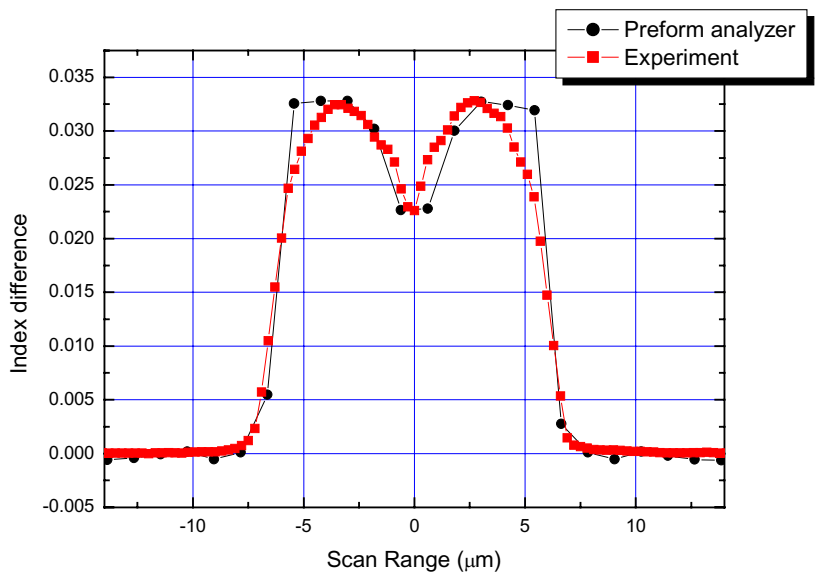

(b)

FIG. 1. (a) Measured refractive index profile of a step index multimode fiber with $13.4 \mu \mathrm{m}$ core size and 0.0328 peak refractive index difference. The measurement scan range is $30 \mu \mathrm{m} \times 30 \mu \mathrm{m}$ with a step size of $300 \mathrm{~nm}$. (b) The comparison between our measurement result for the sample fiber and the refractive index of its preform measured with a conventional preform analyzer.

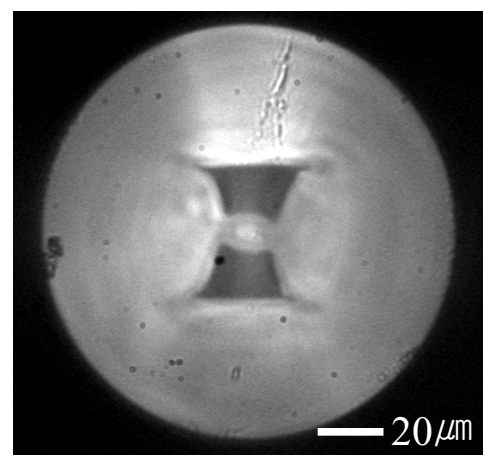

(a)

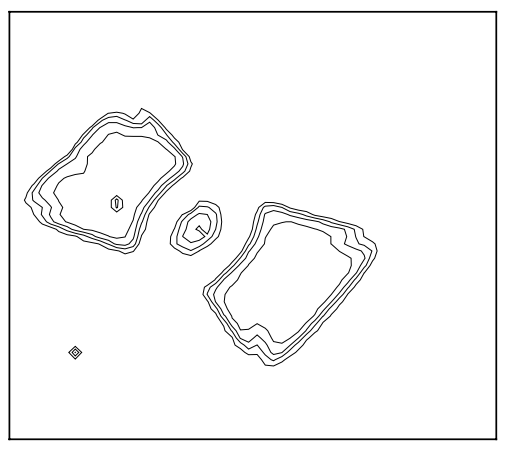

(c)

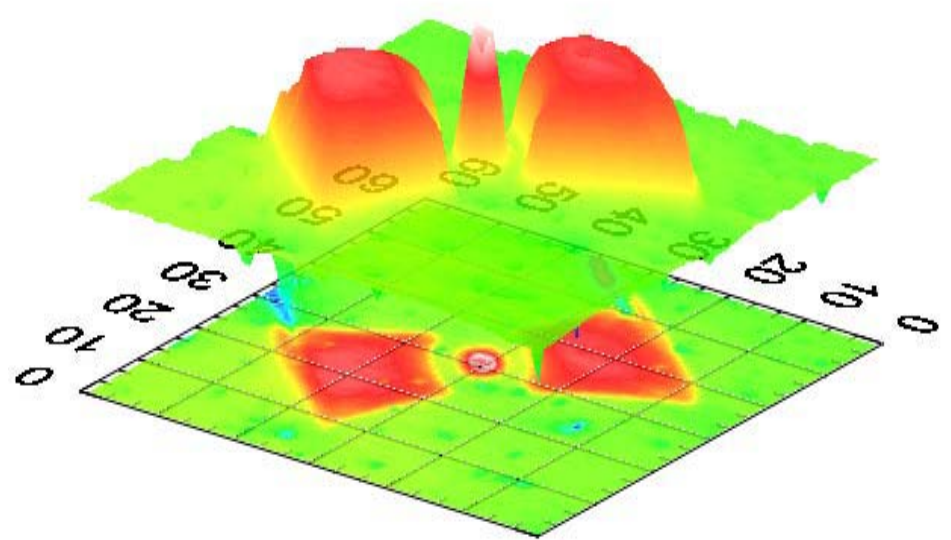

(b)

FIG. 2. (a) The cross-sectional image of a Polarizing Maintaining Fiber (PMF), (b) the measured refractive index profile of a PMF, and (c) its contour plot. 